\title{
GPPS-CH-2020-0033
}

\section{INVESTIGATING THE EFFECTS OF FAN-SHAPED COOLING HOLE GEOMETRY PARAMETERS ON FILM COOLING EFFECTIVENESS FOR INTERNAL COOLANT CROSS-FLOW USING CFD}

\author{
Fotios Gousopoulos \\ Technical University of Crete, School of \\ Production Engineering and Management \\ fgousopoulos@isc.tuc.gr \\ Chania, Crete, Greece
}

\author{
loannis K. Nikolos \\ Technical University of Crete, School of \\ Production Engineering and Management \\ jnikolo@dpem.tuc.gr \\ Chania, Crete, Greece
}

\begin{abstract}
The geometry and distribution of cooling holes play a vital role in the effectiveness of the surface cooling of gas turbine blades, as well as in the design and operation of modern gas turbines. The geometry parameters of a cooling hole affect the downstream temperature and flow fields, as well as the adiabatic film cooling effectiveness distribution. To gain a better view on the way the cooling holes' performance is affected, cross-flow (with internal cooling flow direction normal to the external hot gas flow) CFD simulations were performed for fan-shaped cooling holes. Initially, an existing cooling hole geometry was considered, while then the cooling hole geometry was rotated around the symmetry axis of its cylindrical part. The simulation results revealed that the cooling hole rotation affects adiabatic effectiveness positively at low and intermediate blowing ratios. The observation that the cooling flow attaches to the walls of the rotated holes more effectively is the main reason for the increase in the cooling effectiveness.
\end{abstract}

\section{INTRODUCTION}

Over the years, the efficiency of gas turbines has increased significantly. This increase has been directly linked to the increase in Turbine Inlet Temperature (TIT) (Han et al., 2013). Increasing the TIT to temperatures beyond the melting point of any metal was made possible by adopting various cooling techniques of the turbine blades. One of the most common techniques is film cooling, in which cool air is channelled to the outer surface of the blade through a cooling path, thus creating a cooling layer (Han et al., 2013). The parameters that express the performance of a cooling system are (Maikell et al., 2011)

$$
\begin{gathered}
\varphi=\frac{T_{\infty}-T_{w}}{T_{\infty}-T_{c}} \\
\eta=\frac{T_{\infty}-T_{a w}}{T_{\infty}-T_{c}}
\end{gathered}
$$

where $\varphi$ is overall and $\eta$ is adiabatic effectiveness, $T_{\infty}$ is mainstream temperature, $T_{w}$ is wall temperature, $T_{a w}$ is adiabatic wall temperature and $T_{c}$ is coolant temperature. In (Maikell et al., 2011) they calculated $\varphi$ on the leading edge for various AoA and Biot number $B i=1.8$, while in (Murata et al., 2012) $\eta$ was calculated on the trailing edge area for different blowing ratios $M$, concluding that for $M$ beyond 1.5 there was a slight change in cooling effectiveness.

Most of existing studies in the open literature refer to cylindrical cooling holes' performance and flow field analysis investigations. However, recent studies also investigated shaped holes, which consist of a cylindrical part at the hole's entrance and a diffusing outlet. Shaped holes exhibit different flow phenomena compared to the cylindrical ones. In the cylindrical holes a counter-rotating vortex pair, known as kidney vortices, is developed at the internal, drawing the main flow hot temperature air towards the surface (Bunker et al. 2005). On the contrary, in shaped holes, the kidney vortices effect is reduced through the formation of anti-kidney vortices at the internal of the hole (Haven et al., 1997). Another important flow phenomenon is the separation bubble forming at the diffusing part of the hole (Saumweber et al., 2012a), which causes ingestion of mainstream gas (Kohli et al. 1999; Thole et al., 1998; Lutum et al., 2000; Saumweber et al., 2012b). Shaped holes' cooling effectiveness was found to be optimal for intermediate blowing ratios (Saumweber et al., 2008; Schroeder et al., 2014). This finding does not apply in all cases, though (Saumweber et al., 2012a).

In previous years, several researchers tested cooling holes with a compound injection angle. In (Sen et al., 1996; Schmidt et al., 1996) it was noticed that cylindrical holes with a compound angle improved adiabatic effectiveness and lateral coolant distribution (Brittingham et al., 2000; Dittmar et al., 2003). However, heat transfer levels did also increase. Correspondingly, a compound injection angle also improved 
coolant distribution and adiabatic effectiveness (Haydt et al., 2019). In some studies though it was noticed that hot gas was ingested at the film hole exit (Brittingham et al., 2000). Compared to the cylindrical holes without a compound angle, adiabatic effectiveness was found to be at higher values, especially at high blowing rates (Waye et al., 2007).

Regarding internal cross-flow, film-hole length-todiameter ratio, blowing ratio, and injection angle are the three parameters which affect the flow at the internal of the cooling hole (Leylek et al., 1993). In (Gritsch et al., 2003) it was indicated that for fan-shaped holes film cooling effectiveness was reduced when internal cross-flow was applied; the coolant inclined towards the one side of the diffused part, thus the distribution of the cooling jet was not optimal. In (Saumweber et al., 2012a) the authors concluded that this coolant inclination was influenced by blowing ratio and cooling flow Mach number. In (McClintic et al., 2015; McClintic et al., 2018) geometries with compound injection angles were studied, concluding that higher adiabatic effectiveness was achieved for crossflow counter to the direction of the coolant injection. Finally, it was confirmed that ingestion of mainstream gas was present in cross flow conditions too (McClintinc et al., 2018).

In this work, the cooling effectiveness of a fan-shaped cooling hole is investigated in cross-flow, with and without rotation. Contrary to the previous works on the subject, the rotation of the cooling hole is applied with respect to the axis-of-symmetry of the hole. The objective of this study is to investigate how this rotation around the axis-of-symmetry of the fan-shaped cooling hole affects its cooling effectiveness and the corresponding flow field.

\section{METHODOLOGY}

In this study, a detailed analysis of the cooling efficiency of fan-shaped cooling holes will be presented. The flow field of a fan-shaped cooling hole was simulated with cross-flow present (Fig. 1) at the cooling hole entrance and for different blowing ratios.

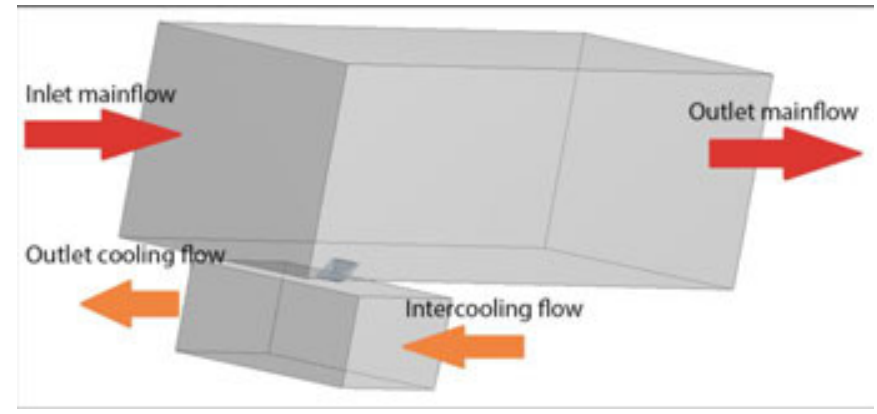

Figure 1 Simulation Domain

Fig. 2 illustrates the geometry of the hole (Saumweber et al., 2008), which is a $14^{\circ}$ laterally expanded hole. The length is $6 D$, the diameter was set to $1.2 \mathrm{~mm}$ and the inclination angle is $30^{\circ}$. Subsequently, it was examined whether the rotation of the hole with respect to the symmetry axis of its cylindrical part (Fig. 3) affects the cooling effectiveness.
Specifically, the original hole was rotated by $15^{\circ}$ and $20^{\circ}$ (Fig. 3).

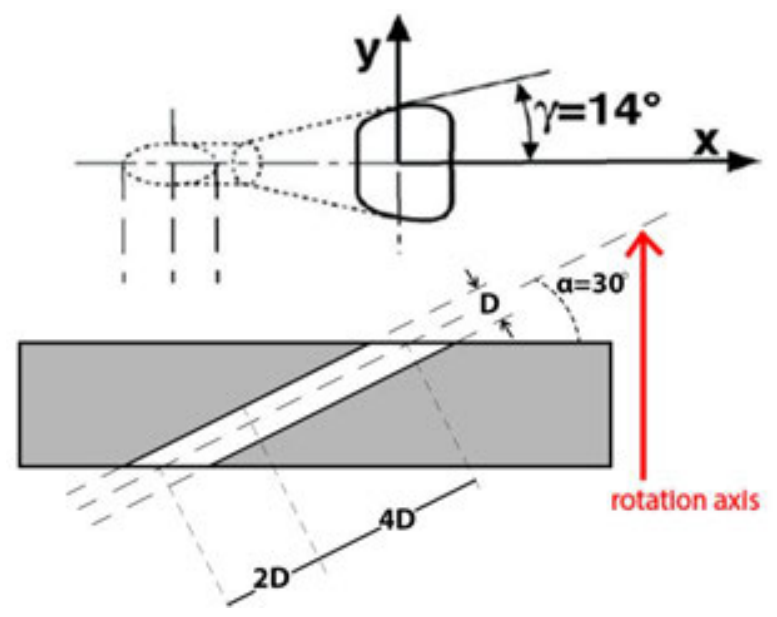

Figure 2 Geometry of the Cooling Hole

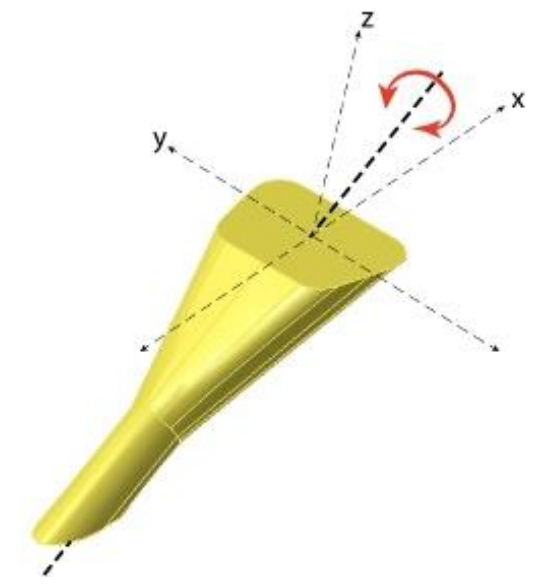

\section{Figure 3 Definition of the Rotation Angle for a Fan- shaped Cooling Hole}

The CFD simulations were utilized using the ANSYS CFX commercial software. The domain's dimensions were $50 D$ upstream the cooling hole, $110 D$ downstream, with $34 D$ width and $35 D$ height for the rectangular domain at the exit of the cooling hole. Correspondingly, a 35D length, $30 \mathrm{D}$ width and $15 D$ height was used for the rectangular domain at the entrance to the cooling hole. A hybrid mesh, consisting of tetrahedral and prismatic elements, was constructed. The final grid consisted of 7,020,168 nodes, 32,954,356 elements for the $0^{\circ}$ case, $9,804,411$ nodes, $49,014,546$ elements for the $15^{\circ}$ case, and 9,873,215 nodes, 49,139,506 elements for the $20^{\circ}$ case (Fig. 4). RANS (Reynolds-Averaged NavierStokes) equations for compressible flow of air (ideal gas) were iteratively solved (steady-state flow), while the SST (Shear Stress Transport) model was used for turbulence modelling.

At the inlet planes of the domain Total Pressure and Total Temperature were specified. At the outlet planes, Static Pressure was specified (Fig. 1). No-slip boundary conditions were imposed on solid surfaces, while at the remaining side planar surfaces free-slip conditions were imposed. 


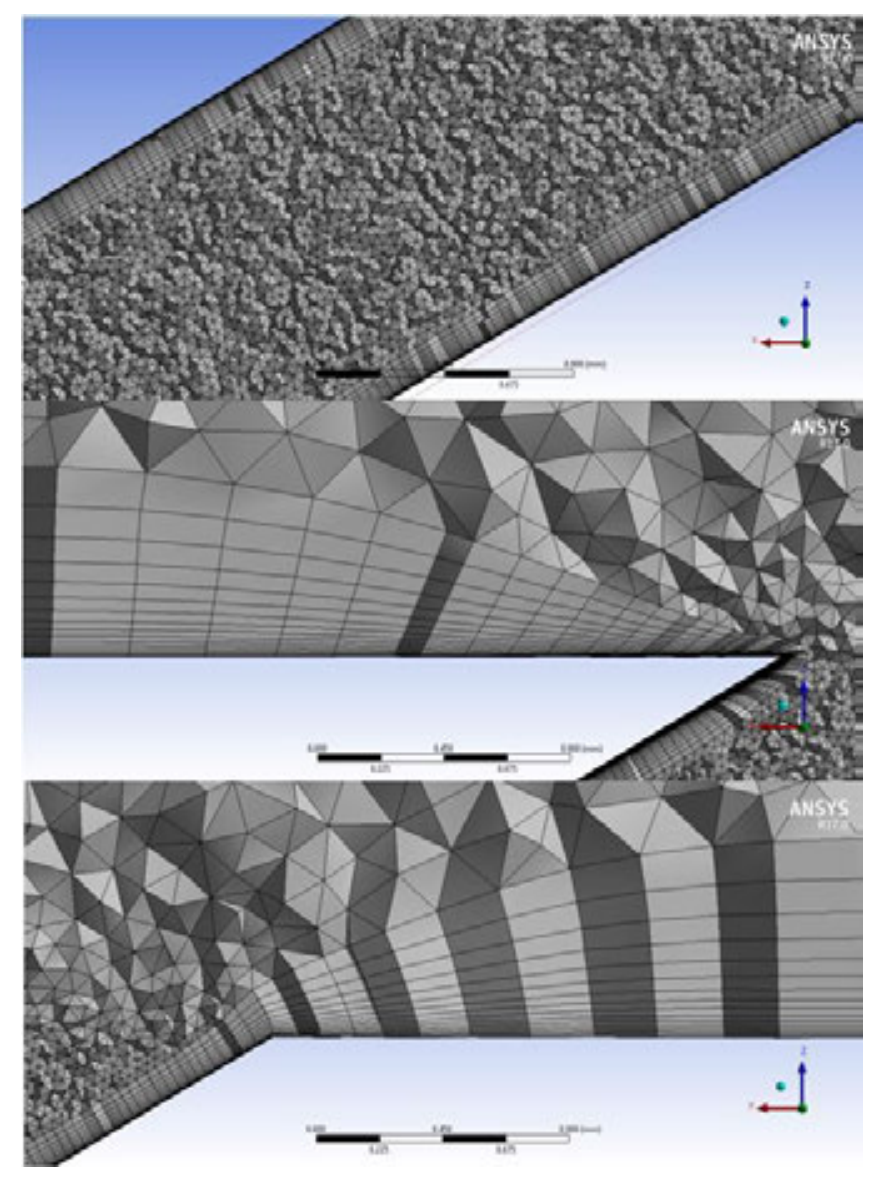

Figure 4 Mesh Details

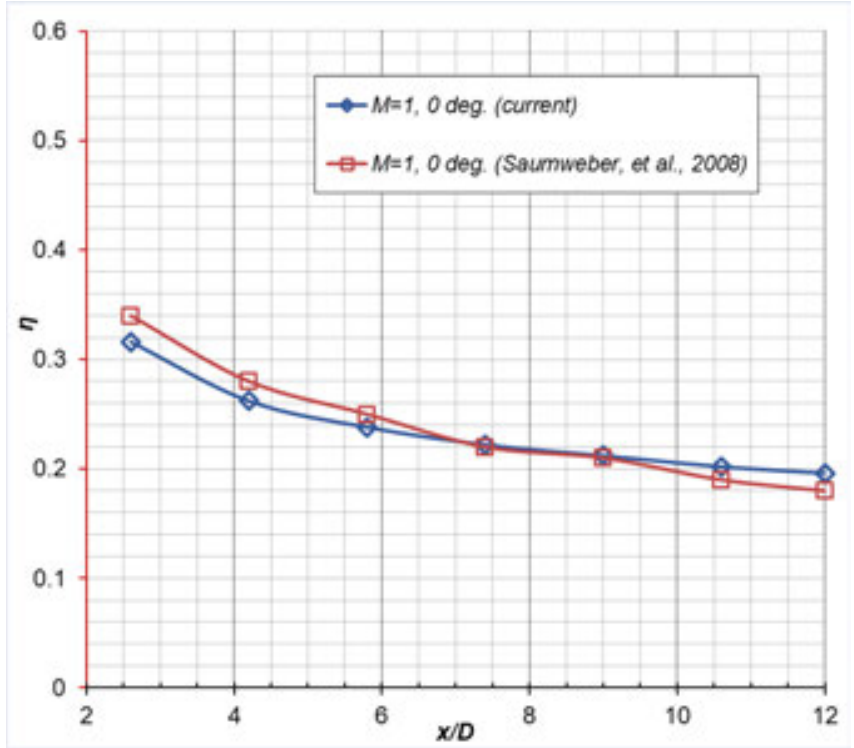

Figure 5 Lateral Adiabatic Effectiveness Comparison, $\mathrm{M}=1,\left(0^{\circ}\right.$ Case $)$

Mainflow density $\rho_{m}$ and velocity $U_{m}$ (average velocity) were evaluated upstream of the cooling hole's exit, $\rho_{c}$ was evaluated at a plane $8 \mathrm{D}$ upstream the entrance to the hole, while $U_{c}$ was evaluated at the hole's exit (mass averaged velocity). The boundary conditions were defined in a way that the desired Mach number in both channels (cooling
$M a_{c}$, and mainflow $M a_{m}$ ), the blowing ratio, $M$, and the density ratio, $D R$, were met. This was achieved through a trial and error procedure. The maximum $y^{+}$for the $0^{\circ}, \mathrm{M}=0.5$ case, was equal to 15 and the minimum equal to 0.0009 , respectively. An automatic blending between a Wall Functions and a Low Reynolds model is used in CFX, allowing for large variations of the $y^{+}$.

Table 1 Boundary Conditions

\begin{tabular}{cccc}
\hline Test case & $\mathbf{1}$ & $\mathbf{2}$ & $\mathbf{3}$ \\
\hline $\boldsymbol{M}$ & 1.00 & 1.50 & 2.50 \\
\hline $\boldsymbol{P}_{s c} / \boldsymbol{P}_{\boldsymbol{m}}$ & 1.06 & 1.13 & 1.34 \\
\hline $\boldsymbol{T}_{s m} / \boldsymbol{T}_{s c}$ & 1.64 & 1.54 & 1.31 \\
\hline $\boldsymbol{T}_{s m}(\mathrm{~K})$ & 1675 & 1675 & 1675 \\
\hline $\boldsymbol{\rho}_{c} / \boldsymbol{\rho}_{\boldsymbol{m}}$ & 1.74 & 1.74 & 1.76 \\
\hline $\boldsymbol{T} u_{m} \%$ & 5.00 & 5.00 & 5.00 \\
\hline $\boldsymbol{T} u_{c} \%$ & 1.00 & 1.00 & 1.00 \\
\hline $\boldsymbol{M} \boldsymbol{a}_{m}$ & 0.30 & 0.30 & 0.30 \\
\hline $\boldsymbol{M} \boldsymbol{a}_{\boldsymbol{c}}$ & 0.29 & 0.29 & 0.29 \\
\hline
\end{tabular}

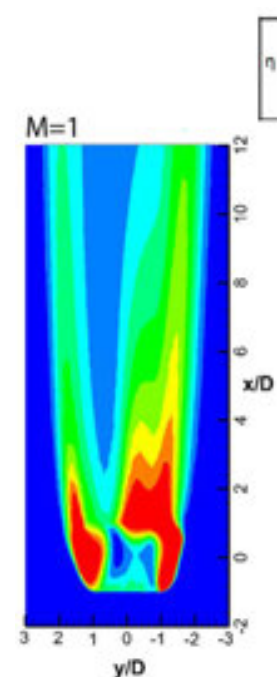

a)
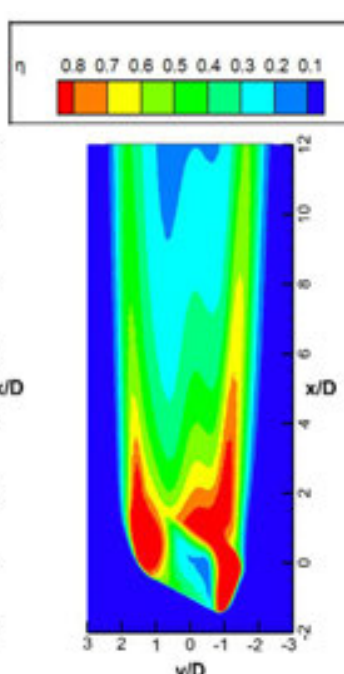

b)

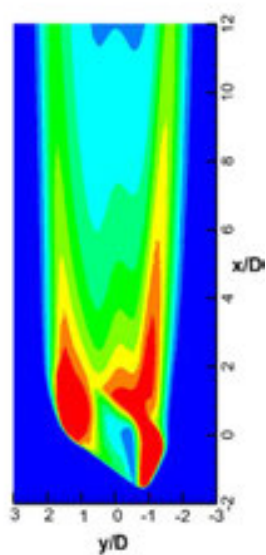

c)
Figure 6 Adiabatic Effectiveness Simulation Results (M=1): a) $0^{\circ}$, b) $15^{\circ}$, c) $20^{\circ}$

The RMS residual target for the iterative solution procedure was set to $10^{-5}$. The final boundary conditions are shown in Table 1. The numerical results were validated by comparing the adiabatic effectiveness measurements $(M=$ 1.0, mainflow Mach number $M a_{m}=0.3$, cooling flow Mach number $M a_{c}=0$ ) to the experimental results from (Saumweber, et al., 2008). An average deviation of 5\% was calculated between the two graphs in Fig. 5.

The adiabatic effectiveness graphs were calculated by measuring the lateral $(-3 \leq y / D \leq 3)$ average adiabatic effectiveness at locations $x / D=2.6,4.2,5.8,7.4,9,10.6$ and 12. In order to compute the blowing ratio, the mass averaged coolant velocity $u_{c}$ was evaluated at the cooling hole exit. Main flow velocity was measured at a location $49 D$ downstream of the inlet mainflow surface. Cooling flow 
static pressure $P_{s c}$ and density $\rho_{c}$ were measured at a location $11 D$ upstream of the hole entrance, while main flow $P_{s m}$ and $\rho_{m}$ at a location $8 D$ upstream of the hole exit.

\section{RESULTS AND DISCUSSION}

\section{Adiabatic Effectiveness Results}

Adiabatic effectiveness contours are shown in Fig. 6, 7 and 8 . Initially, regarding the $0^{\circ}$ case, it can be observed that cooling effectiveness increases with increasing the blowing ratio up to $M=1.5$. Beyond that value, effectiveness is reduced again $(M=2.5)$. In Fig. 9,10 and 11 the temperature distributions, at position $3 D$ downstream the cooling hole exit, show an asymmetric cooling profile.

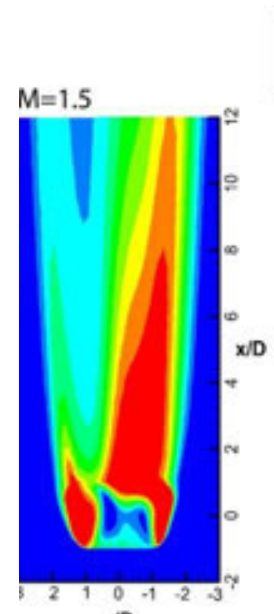

y/D

a)

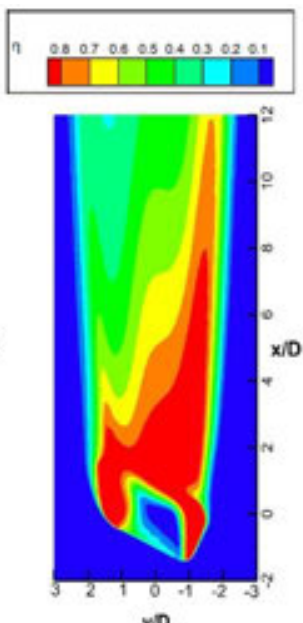

b)

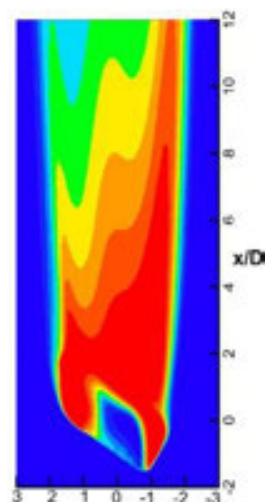

y/D

c)
Figure 7 Adiabatic Effectiveness Simulation Results (M=1.5): a) $0^{\circ}$, b) $15^{\circ}$, c) $20^{\circ}$

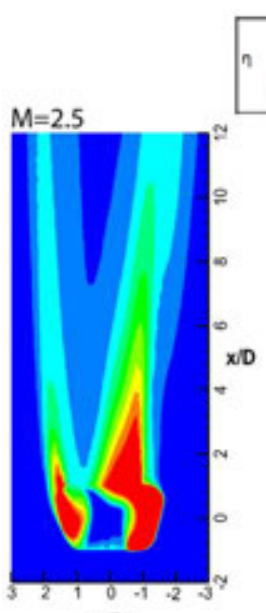

y/o
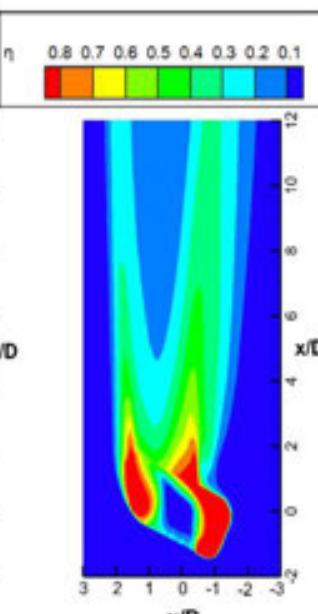

b)

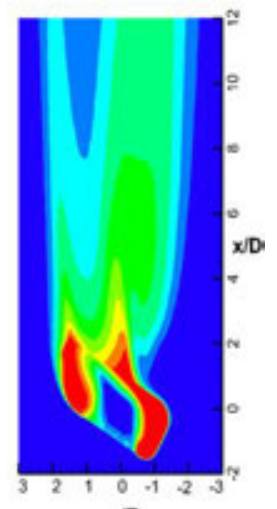

c)
Figure 8 Adiabatic Effectiveness Simulation Results (M=2.5): a) $0^{\circ}$, b) $15^{\circ}$, c) $20^{\circ}$

The cooling layer is enhanced at the side walls of the hole. At the same time, a thinning at the center can also be observed, hence the observed distribution profiles in Fig. 6, 7 and 8. If normal cross-flow exists at the hole entrance (as in our case) a single vortex is formed inside the hole, leading to a non-symmetric inflow into the diffusing part of the hole and to a reduced film cooling effectiveness (Saumweber, et al, 2008); however, cross-flow causes a deeper penetration of the coolant into the hot gas, without strong lift-off. According to (Saumweber, et al, 2008), the fan-shaped holes demonstrate a negative impact of the cross-flow, especially at high blowing rates $(\mathrm{M}=2.5)$, which was also confirmed in this study. The cooling jet lift-off at $M=2.5$ is observed clearly in Fig. 11.

a) $\frac{\mathrm{N}}{\mathrm{N}}$

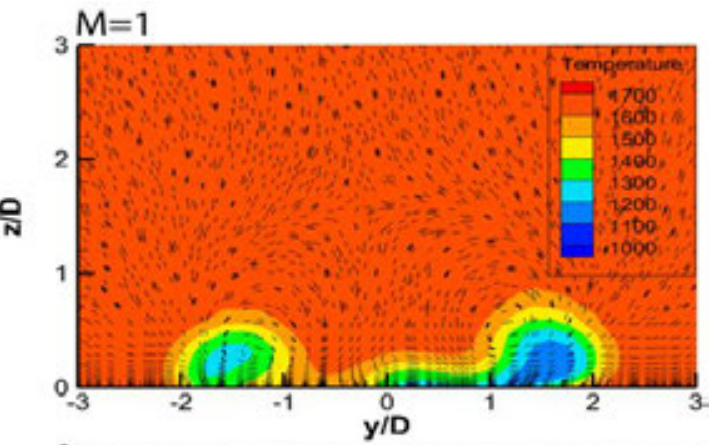

b) $\frac{\cap}{N}$
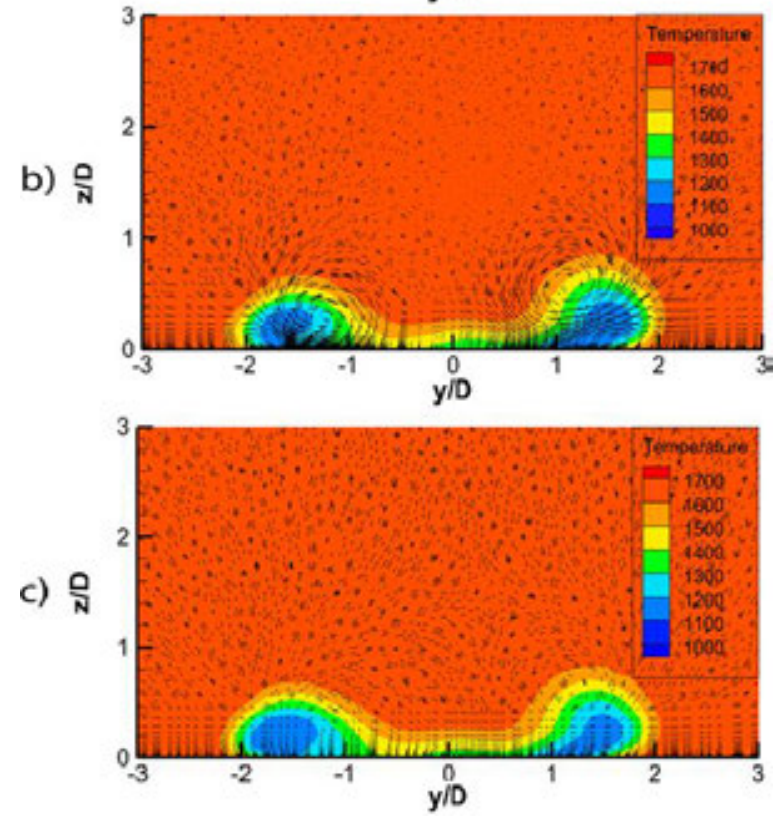

Figure 9 Calculated Velocity Field and Gas Temperature at a Position 3D Downstream the Cooling Hole $(M=1)$ : a) $0^{\circ}$, b) $15^{\circ}$, c) $20^{\circ}$

The simulation results for the two geometries created by rotating the cooling hole by $15^{\circ}$ and $20^{\circ}$, showed an increase in adiabatic effectiveness. The graph in Fig. 12 clearly reveals that the rotation increased the adiabatic effectiveness. Specifically, for a low blowing ratio $(M=1)$, there is an increase in effectiveness, $12 \%$ in average for the $15^{\circ}$ case and $18 \%$ for the $20^{\circ}$ case. It is also notable that for locations near the hole's exit (up to $x / D=6$ from the hole's exit) the increase in adiabatic effectiveness is even higher. For blowing ratio $M=1.5$, the increase in effectiveness is even higher. The difference in effectiveness, which can be easily revealed in the corresponding graphs, translates to $55 \%$ and $61 \%$ increase for the $15^{\circ}$ case and $20^{\circ}$ case, respectively. Despite the fact that at low and intermediate 
blowing ratios the performance increases, at high ratios the opposite phenomenon is observed (as expected). For $M=$ 2.5 a decrease in effectiveness of about $12 \%$ for the $15^{\circ}$ case is observed (compared to the $0^{\circ}$ case), with a slight increase of $7.5 \%$ for the $20^{\circ}$ case.

a)

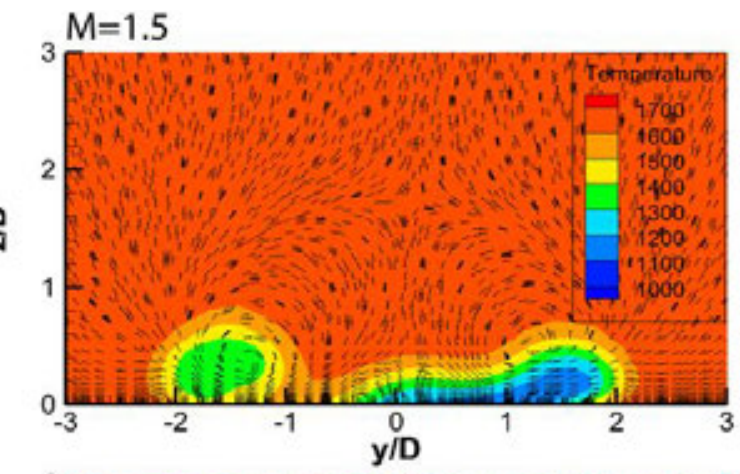

b) $\frac{\rho}{\mathrm{N}}$

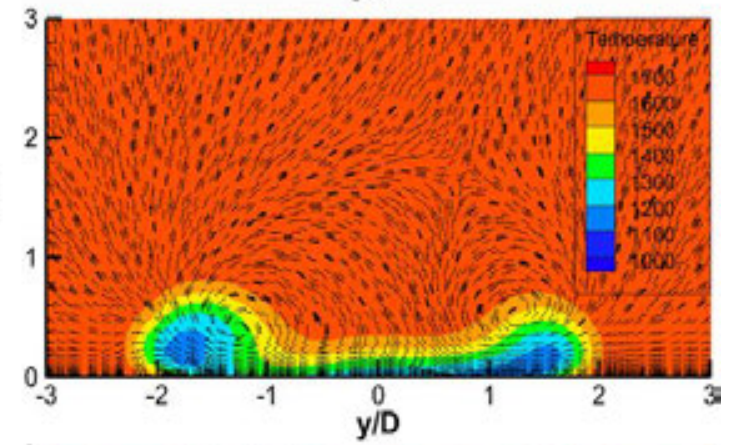

c) $\frac{\mathrm{N}}{\mathrm{N}}$

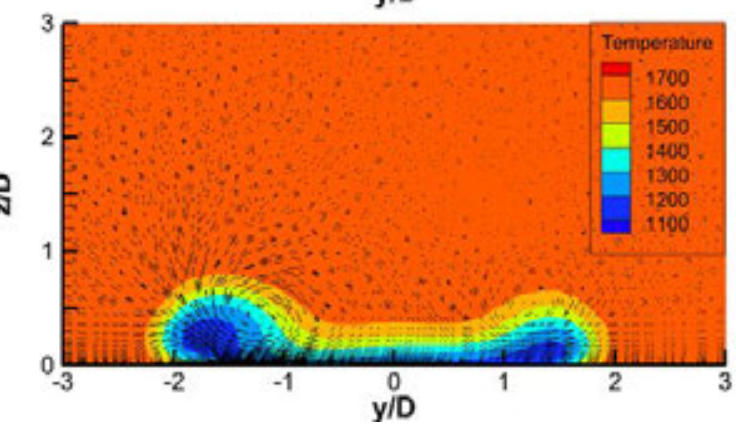

Figure 10 Calculated Velocity Field and Gas Temperature at a Position 3D Downstream the Cooling Hole $(\mathrm{M}=1.5)$ : a) $0^{\circ}$, b) $15^{\circ}$, c) $20^{\circ}$

Through the rotation of the cooling hole the cooling jet penetrates through the main flow walls without lift-off (Fig. $9,10)$, hence the adiabatic effectiveness grows. While in the $0^{\circ}$ case the coolant detachment is initiated at the locations near the cooling hole, the flow for the rotated cooling hole (at both $15^{\circ}$ and $20^{\circ}$ cases) seems to reduce that detachment. The above observations apply for $M=1$ and $M=1.5$. At $M=2.5$, jet lift-off remains almost unchanged.

In general, the cooling hole rotation affects adiabatic effectiveness positively at low and intermediate blowing ratios. The fact that the cooling flow attaches to the walls more effectively is the main reason for the observed increase in the cooling effectiveness. Additionally, the width of the cooling layer is also expanded. However, at high blowing ratios, the effectiveness is maintained at similar levels to the $0^{\circ}$ case.
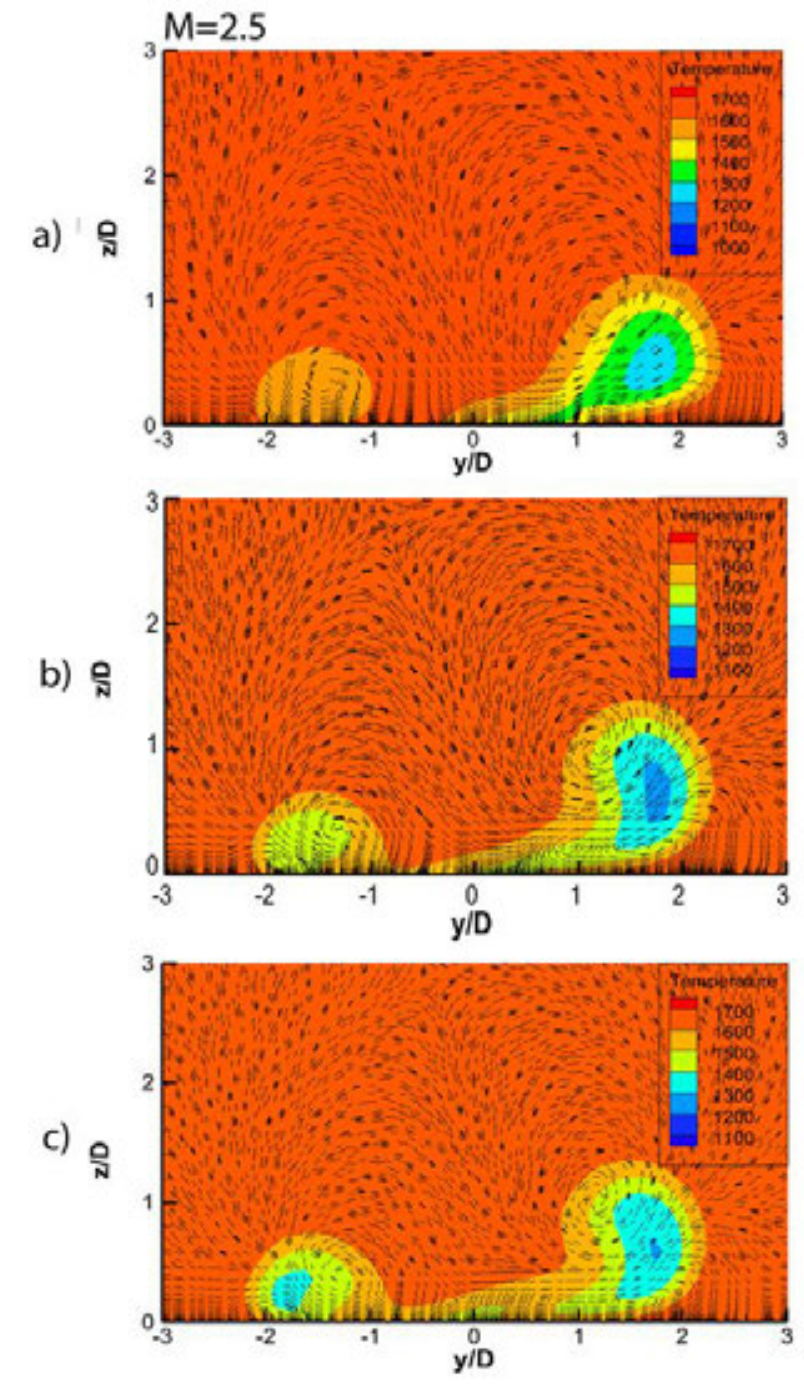

Figure 11 Calculated Velocity Field and Gas Temperature at a position $3 D$ Downstream the Cooling Hole $(M=2.5)$ : a) $0^{\circ}$, b) $15^{\circ}$, c) $20^{\circ}$

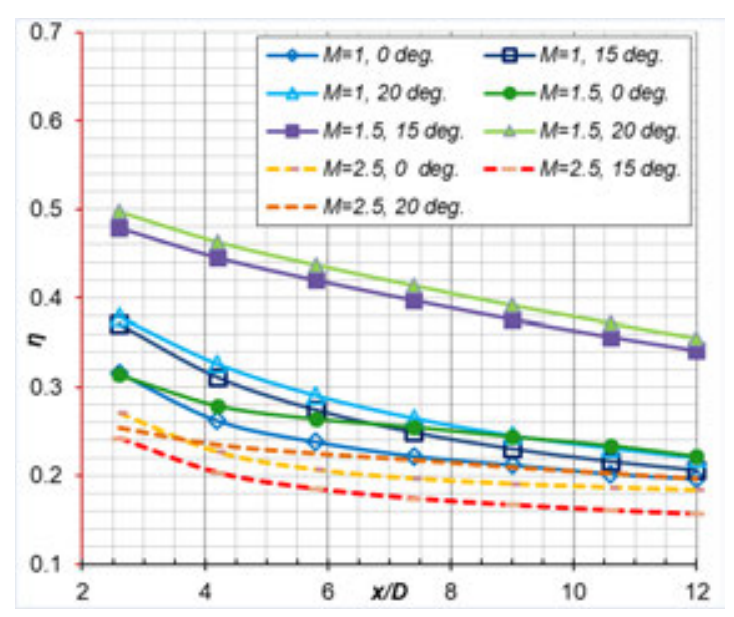

Figure 12 Effect of Coolant Cross-flow on Laterallyaveraged Film Cooling of Rotated Fan-shaped Holes 

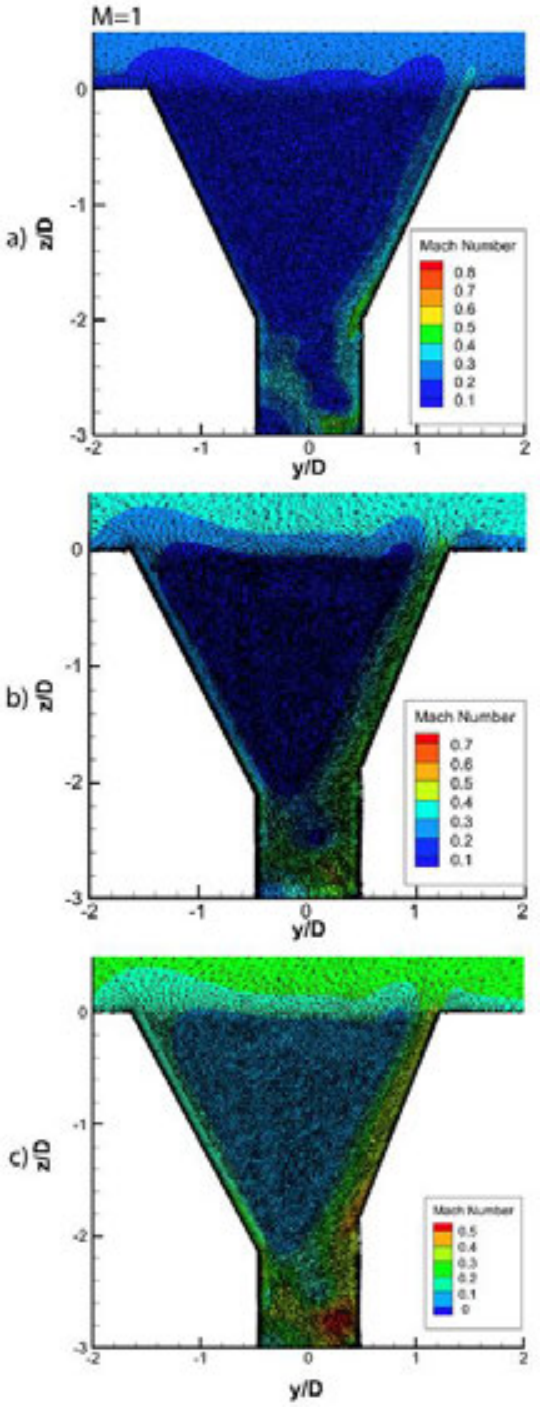

\section{Figure 13 Contours of Mach Number at the Interior of the Cooling Hole $(M=1)$ : a) $0^{\circ}$, b) $15^{\circ}$ and c) $20^{\circ}$}

\section{Flow field inside the cooling hole}

The flow field inside the cooling hole is analysed to comprehend why the blowing ratio and the rotation angle affect cooling efficiency. Fig. 13, 14 and 15 show the Mach number at a plane that is perpendicular to the symmetry plane of the cooling hole and passes through the cylinder's axis. It is observed that the position where the vortex is formed is affected neither by the hole rotation nor by the alteration of the blowing ratio. However its strength is clearly affected by the blowing ratio. Regarding the alteration of the blowing ratio, it is observed that the vortex that initiates in the cylindrical part of the cooling hole (approximately at level $z / D=-2.4$ ) expands when entering the diffusing part of the hole. As a result, the air acquires higher velocity near the side wall and low velocity at the central region of the cooling hole.

As the blowing ratio increases, so does the velocities inside the cooling hole. However, due to the flow's strong non-uniformity, the velocity that eventually develops on the walls can be reduced (due to the applied rotation, which smoothens the exit flow). For example, at $M=1.5$, although the initial value of the velocity is high, it decreases at the exit of the cooling hole and therefore the cooling effectiveness increases. However, at $M=2.5$ the exit Mach number at the side wall remains high, along with the flow non-uniformity, despite the applied rotation.

a)

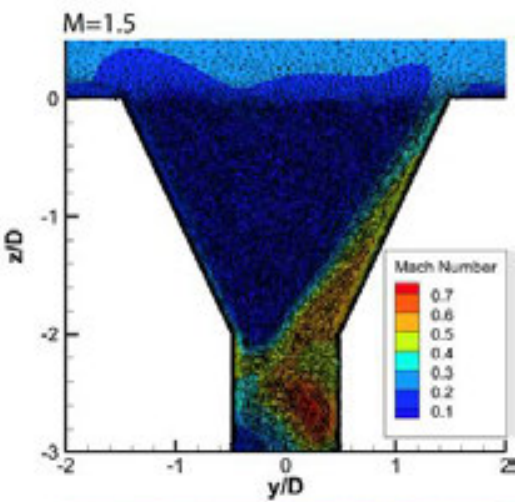

b)
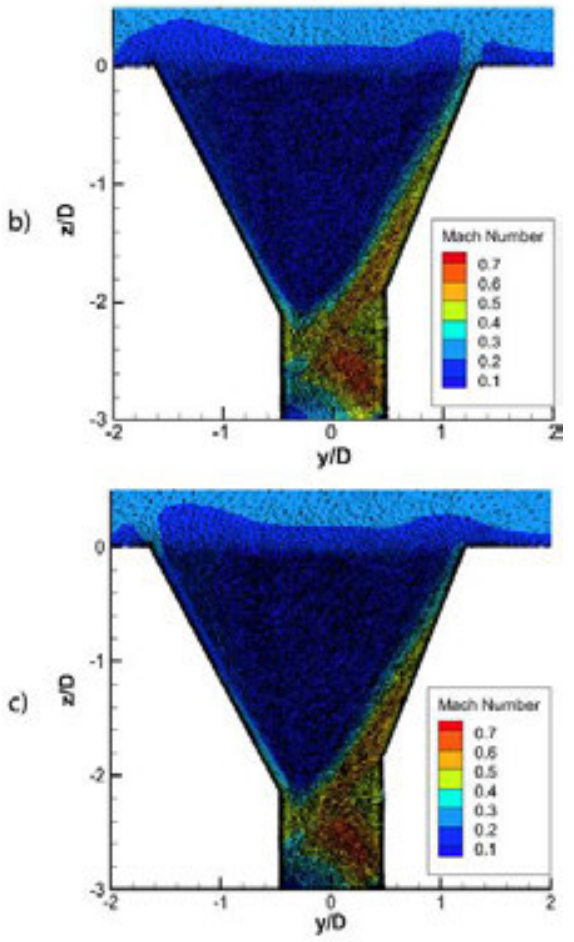

Figure 14 Contours of Mach Number at the Interior of the Cooling Hole (M=1.5): a) $0^{\circ}$, b) $15^{\circ}$ and c) $20^{\circ}$

For better understanding the effect of rotating the cooling hole, parallel planes were defined (Fig. 16) where the Mach number is displayed, for $M=1.5$. The value of $M=$ 1.5 was chosen since, for this value, the changes in effectiveness due to rotation of the cooling hole are the most noticeable. The comparison between the 3 considered cases is shown in Fig. 17.

At the cylindrical part of the hole the flow remains almost unchanged with the applied rotation, as opposed to the diffusing part. The applied rotation seems to help the flow to become more uniform in the lateral direction, inside the diffusing part of the hole. Thus, the formed cooling layer remains attached to the walls when exiting the hole. 
For completeness, a grid independence study is presented here, for the $0^{\circ}, \mathrm{M}=0.5$ case. Three grids were used, having around 2.5, 5.0 and 7.0 million nodes respectively. The results (for the three grids) of the adiabatic effectiveness distribution are depicted in Fig. 18.
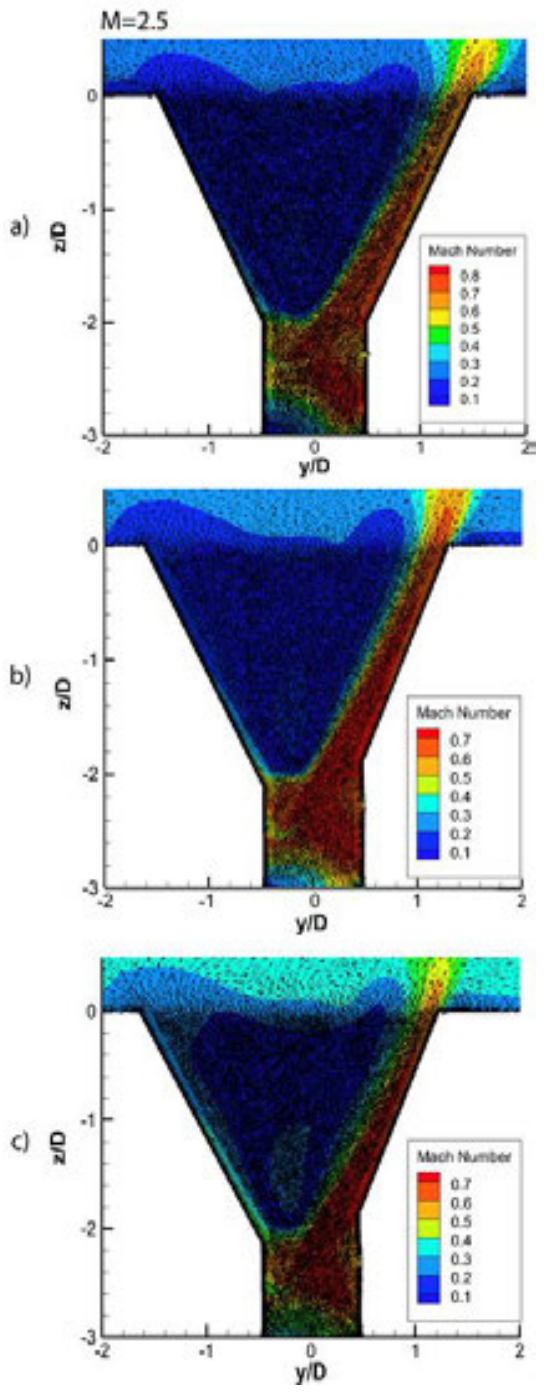

Figure 15 Contours of Mach Number at the Interior of the Cooling Hole (M=2.5): a) $0^{\circ}$, b) $15^{\circ}$ and c) $20^{\circ}$

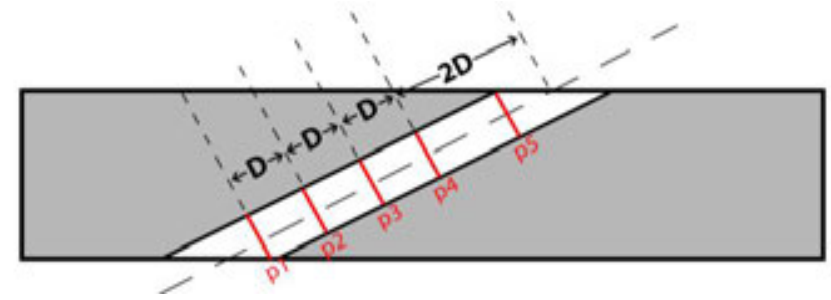

Figure 16 Planes p1, p2, p3, p4, p5

\section{CONCLUSIONS}

The objective of this study was to investigate how the rotation of a fan-shaped cooling hole around the symmetry axis of its cylindrical part affects the cooling efficiency. It was revealed that, at low and intermediate blowing ratios, the rotation assisted the uniformity of the high velocity layer at the exit of the cooling hole. Consequently, the distribution of the coolant was more effective, resulting in an increase to the cooling effectiveness. Nonetheless, the applied rotation did not appear to have similarly positive effect at high blowing ratios. The effect of negative rotation angles on the cooling effectiveness is under investigation.
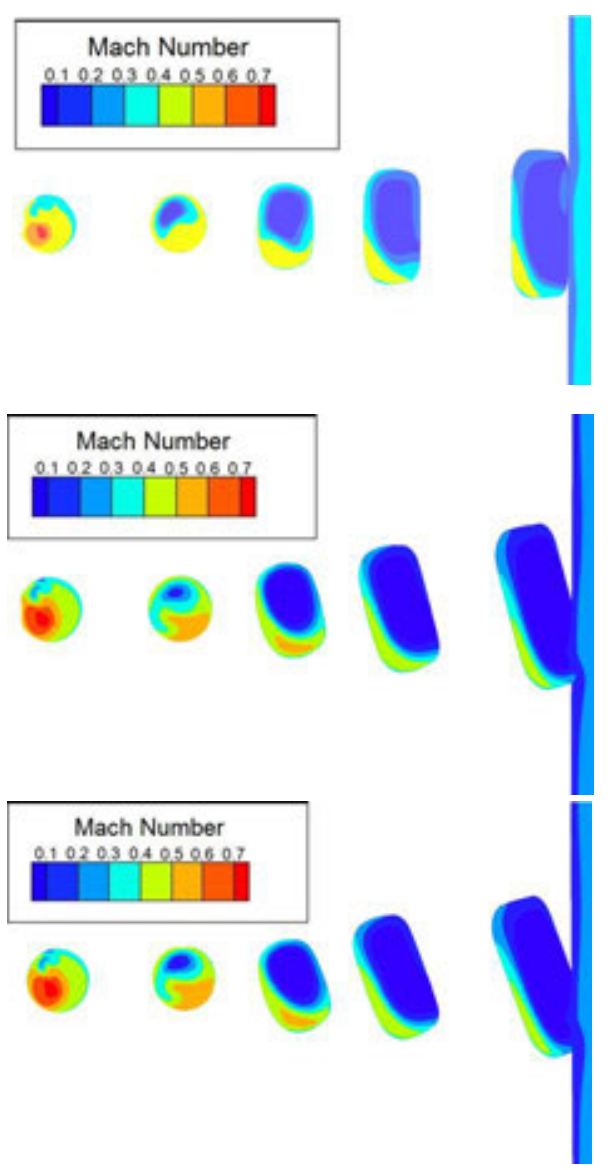

Figure 17 Contours of Mach Number at Planes p1, p2, p3, p4, p5 (from top: $\left.0^{\circ}, 15^{\circ}, 20^{\circ}\right)(M=1.5)$

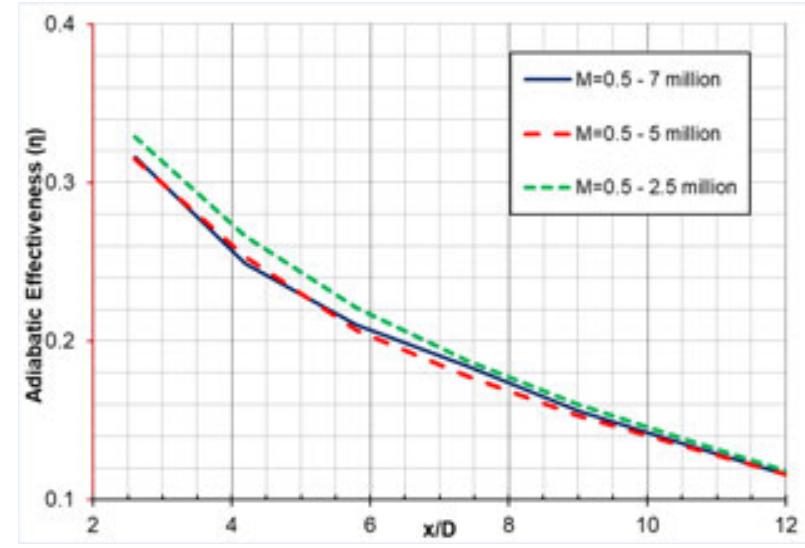

Figure $18 \mathrm{Grid}$ Independence Test Results (0, $M=0.5$ )

\section{NOMENCLATURE}

Bi Biot Number

D Film cooling hole diameter

DR Density ratio 


$\begin{array}{ll}\mathrm{M} & \text { Blowing ratio } \\ \mathrm{Ma} & \text { Mach number } \\ \mathrm{P} & \text { Pressure } \\ \mathrm{T} & \text { Temperature } \\ \mathrm{TIT} & \text { Turbine Inlet Temperature } \\ \mathrm{Tu} & \text { Turbulence intensity } \\ \mathrm{U} & \text { Velocity } \\ \mathrm{x} & \text { streamwise distance } \\ \mathrm{y} & \text { lateral distance } \\ \mathrm{z} & \text { normal distance }\end{array}$

\section{Greek Symbols}

$\begin{array}{ll}\eta & \text { Adiabatic effectiveness } \\ \rho & \text { Density } \\ \varphi & \text { Cooling effectiveness }\end{array}$

$\begin{array}{ll}\text { Subscripts } \\ \text { aw } & \text { Adiabatic wall } \\ \mathrm{c} & \text { Cooling flow } \\ \mathrm{m} & \text { Mainflow } \\ \mathrm{sc} & \text { Static, cooling flow } \\ \mathrm{sm} & \text { Static, mainflow } \\ \text { tc } & \text { Total, cooling flow } \\ \mathrm{tm} & \text { Total, mainflow }\end{array}$

\section{REFERENCES}

Brittingham, R., Leylek, J., (2000). A Detailed Analysis of Film Cooling Physics: Part IV - Compound-Angle Injection With Shaped Holes. ASME Journal of Turbomachinery, 122(1), pp. 133-145, doi: 10.1115/1.555419.

Bunker, R. (2005). A Review of Shaped Hole Turbine Film Cooling Technology. Journal of Heat Transfer, 127(4), pp. 441-453, doi: 10.1115/1.1860562.

Dittmar, J., Schulz, A., Wittig, S., (2003). Assessment of Various Film-Cooling Configurations Including Shaped and Compound Angle Holes Based on Large-Scale Experiments. ASME Journal of Turbomachinery, 125(1), pp. 57-64, doi: 10.1115/1.1515337.

Han, J.-C. (2013). Fundamental Gas Turbine Heat Transfer. ASME Journal of Thermal Science and Engineering Applications, 5(2), 021007, doi: 10.1115/1.4023826.

Haven, B., Yamagata, D., Kurosaka, M., Yamawaki, S. and Maya, T. (1997). Anti-Kidney Pair of Vortices in Shaped Holes and Their Influence on Film Cooling Effectiveness. In: ASME International Gas Turbine and Aeroengine Congress, 97-GT-45, V003T09A007, Orlando, Florida, USA, June 2-5, doi: 10.1115/97-GT-045.

Haydt, S., Lynch, S., (2019). Cooling Effectiveness for a Shaped Film Cooling Hole at a Range of Compound Angles. ASME Journal of Turbomachinery, 141(4), 041005, doi: 10.1115/1.4041603.

Kohil, A. and Bogard, D. (1999). Effects of Hole Shape on Film Cooling With Large Angle Injection. In: ASME International Gas Turbine and Aeroengine Congress and Exhibition, 99-GT-165, V003T01A04, Indianapolis, Indiana, USA, June 7-10, doi: 10.1115/99-GT-165.

Leylek, J., Zerkle, R., (1994). Discrete-Jet Film Cooling: A Comparison of Computational Results With Experiments.
ASME Journal of Turbomachinery, 116(3), pp. 358-368, doi: 10.1115/1.2929422.

Lutum, E., von Wolfersdorf, J., Weigand, B., and Semmler, K., (2000). Film Cooling on a Convex Surface with Zero Pressure Gradient Flow. International Journal of Heat and Mass Transfer, 43(16), pp. 2973- 2987, doi: 10.1016/S00179310(99)00346-4.

Maikell, J., Bogard, D., Piggush, J. and Kohli, A. (2011). Experimental Simulation of a Film Cooled Turbine Blade Leading Edge Including Thermal Barrier Coating Effects. ASME Journal of Turbomachinery, 133(1), 011014, doi: 10.1115/1.4000537.

McClintic, J., Klavetter, S., Winka, J., Anderson, J., Bogard, D., Dees, J., Laskowski, G., Briggs, R., (2015). The Effect of Internal Crossflow on the Adiabatic Effectiveness of Compound Angle Film Cooling Holes. ASME Journal of Turbomachinery, 137(7), 071006, doi: 10.1115/1.4029157.

McClintic, J., Anderson, J., Bogard, D., Dyson, T., Webster, Z., (2018). Effect of Internal Crossflow Velocity on Film Cooling Effectiveness-Part II: Compound Angle Shaped Holes. ASME Journal of Turbomachinery, 140(1), 011004, doi: 10.1115/1.4037998.

McClintic, J., Fox, D., Jones, F., Bogard, D., Dyson, T., Webster, Z., (2018). Flow Physics of Diffused-Exit Film Cooling Holes Fed by Internal Crossflow. ASME Journal of Turbomachinery, 141(3), 031010, doi: 10.1115/1.4042166.

Murata, A., Nishida, S., Saito, H., Iwamoto, K., Okita, Y. and Nakamata, C. (2012). Effects of Surface Geometry on Film Cooling Performance at Airfoil Trailing Edge, ASME Journal of Turbomachinery, 134(5), 051033, 051033, doi: 10.1115/1.4004828.

Saumweber, C. and Schulz, A. (2008). Comparison of the Cooling Performance of Cylindrical and Fan-Shaped Cooling Holes with Special Emphasis on the Effect of Internal Coolant Cross-Flow. In: ASME Turbo Expo 2008: Power for Land, Sea, and Air, Berlin, Germany, June 9-13, doi: 10.1115/GT2008-51036.

Saumweber, C. and Schulz, A., (2012). Free-stream Effects on the Cooling Performance of Cylindrical and Fan-shaped Cooling Holes. ASME Journal of Turbomachinery, 134(6), 061008, doi: 10.1115/1.4006290.

Schmidt, D., Sen, B., Bogard, D., (1996). Film Cooling With Compound Angle Holes: Adiabatic Effectiveness. ASME Journal of Turbomachinery, 118(4), pp. 807-813, doi: 10.1115/1.2840938.

Sen, B., Schmidt, D., Bogard, D., (1996). Film Cooling With Compound Angle Holes: Heat Transfer. ASME Journal of Turbomachinery, 118(4), pp. 800-806, doi: 10.1115/1.2840937.

Thole, K., Gritsch, M., Schulz, A., and Wittig, S., (1998). Flowfield Measurements for Film Cooling Holes With Expanded Exits. ASME Journal of Turbomachinery, 120(2), pp. 327-336, doi: 10.1115/1.2841410.

Waye, S., Bogard, D., (2007). High-Resolution Film Cooling Effectiveness Comparison of Axial and Compound Angle Holes on the Suction Side of a Turbine Vane. ASME Journal of Turbomachinery, 129(2), pp. 202-211, doi: 10.1115/1.2448016. 